\title{
Valores Culturales y Consecuencias Psicosociales del Desempleo en América Latina
}

\section{Culture Values and Psycho-social Effects of Unemployment in Latin-America}

\author{
Tomás Izquierdo \\ Universidad de Murcia
}

\author{
Horacio Jorge Alonso \\ Universidad Nacional de La Plata
}

\begin{abstract}
Resumen. Usando una aproximación cualitativa, en este artículo se exploraron las actitudes hacia el trabajo de una muestra integrada por 162 personas desempleadas del interior de Paraguay. Los resultados mostraron que los valores culturales condicionan las actitudes hacia la búsqueda de empleo. Se concluye con propuestas de intervención y futuras investigaciones en el área.

Palabras clave: desempleo, cultura, inserción laboral, personalidad, valores.
\end{abstract}

\begin{abstract}
In this article, using a qualitative approach, attitudes toward work were explored using a sample consisted of 162 unemployed people who live in the interior of Paraguay. The results showed that cultural values affect attitudes toward job search. The work concludes with proposals for intervention and future research in the area.

Key words: unemployment, culture, personality, values.
\end{abstract}

La cultura de una sociedad puede entenderse como un conjunto de valores ubicados a lo largo de ciertas dimensiones, algunas de las cuales parecen ser universales y la ubicación de las personas en cada una de esas dimensiones describe su patrón cultural (Oyserman, Kemmelmeir y Coon, 2002). Hofstede (1984) fue uno de los primeros autores en identificar los valores propios de cada sociedad. Este autor sugirió que las culturas pueden ser clasificadas según su posición en cuatro dimensiones básicas: individualismo-colectivismo, distancia del poder, evitación de la incertidumbre y masculinidad-feminidad.

La influencia de la cultura en las actitudes sobre el trabajo de las personas desempleadas, mediante el desarrollo de una serie de creencias y comportamientos relacionados con la búsqueda de empleo, es un tema que apenas ha sido investigado. Estar sin trabajo se convierte en un obstáculo que dificulta el potencial desarrollo de los individuos impidiendo, de una u otra forma, el acceso de la población a los satisfactores de su bienestar psicológico. Según la tasa de participación de la población activa en 2009, como uno de los indicadores claves de la Organización Internacional del Trabajo (OIT), se está produciendo un incremento de las tasas de desempleo en América Latina, síntoma de los efectos de una crisis internacional sin precedentes que ensombrece la economía y afecta a los países de la región. Esta situación se evidencia en muchos países y, entre ellos en Paraguay, donde el desempleo ha creci-

La correspondencia sobre este artículo debe dirigirse al primer autor al Departamento de Métodos de Investigación, Universidad de Murcia, Campus de Espinardo, 30100 Murcia. E-mail: tomasizq@um.es do en los últimos años, por un lado por los importantes cambios tecnológicos, principalmente las tecnologías de la información y comunicación, que sufren las empresas que les obligan a reducir la mano de obra y, por otro, la recesión económica ha sido fuerte y prolongada lo que ha dejado a mucha gente sin trabajo. En relación con esto, Blanch (2007) ha caracterizado el mercado laboral global, latinoamericano y europeo, como un entorno laboral complejo y dinámico, donde se combinan y confunden diversos tipos y tasas de empleo y desempleo, de trabajo sumergido y subempleo, así como de situaciones de déficit estructural de puesto de trabajo y de déficit individual de capacidades para trabajar en los puestos ofertados.

Según sea el origen que lo provoca, el desempleo afecta a diferentes clases de personas. Por un lado a personas con un cierto nivel de cualificación que son obligadas a abandonar sus trabajos por las sucesivas reformas; por otro, a personas con un nivel muy bajo de formación que pierden su relación laboral en diversos servicios por la situación recesiva. En ambos casos, siguiendo la aportación de Schlemenson (2002), el individuo desplazado del ámbito del trabajo se considera excluido de la sociedad, generándose determinados comportamientos que, en muchas ocasiones, dificultan la puesta en marcha de determinados planes de acción que favorezcan la inserción o reinserción laboral de las personas desempleadas. Sobre la base de lo expuesto, planteamos como objetivo de estudio el análisis de la influencia que los valores culturales ejercen sobre las actitudes hacia el trabajo. De esta manera, la presente investigación trata de evaluar la relevancia de los valores culturales como variable moderadora de los 
efectos del desempleo en contextos hasta la fecha no explorados. Con este propósito, en los siguientes apartados se lleva a cabo una revisión de la literatura sobre las consecuencias psicosociales del desempleo, la influencia de las culturas nacionales y regionales y se culmina con el concepto resiliencia y, posteriormente, se describe un estudio empírico que permitió recopilar 162 entrevistas de personas desempleadas para analizar sus actitudes hacia la búsqueda de empleo.

\section{Consecuencias psicosociales del desempleo}

El desempleo, como fenómeno psicosocial, afecta a diferentes clases de personas y tiene una incidencia considerable en los distintos aspectos de la existencia humana. La falta acusada de puestos de trabajo, asociado como problema principal de la economía y la democracia de los países de América del Sur, se torna más visible en diversas estrategias como el trabajo informal urbano y el subempleo rural, ocultando, en cierta manera, este problema (Izquierdo, 2007). Estas y otras estrategias forman parte de la denominada "cultura del desempleo". El trabajo informal urbano y el subempleo rural comparten con otras situaciones laborales no sólo la precariedad laboral sino también la psicológica, es el caso del empleo temporal involuntario como se muestra en el estudio de Blanch y Cantera (2009). La precariedad laboral, o aún más la falta de un empleo, no solo merma el acceso a las diversas fuentes de obtención de recursos, sino que genera una serie de aspectos negativos sobre el bienestar psicológico de las personas que lo sufren. De acuerdo a García y García (2008) y Garrido (2006), la experiencia psicológica del desempleo varía en función de una serie de variables como la clase social, el género, el nivel de estudios, la edad, el apoyo social o la duración del periodo de desempleo, que determinan una actitud diferenciada hacia el trabajo de las personas en situación de desempleo. Éstas variables se acentúan en aquellos colectivos con mayores dificultades de inserción laboral. En el estudio de Izquierdo (2007), se analizaron las características sociales y psicológicas que repercuten en las probabilidades de encontrar trabajo en uno de éstos colectivos con mayor dificultad como es el caso de los desempleados mayores de 45 años. Otros autores se han centrado en investigar las consecuencias generadas de la situación de desempleo centrándose fundamentalmente en el análisis de las actitudes o la identidad social de los desempleados. En este sentido, por ejemplo, Bauder (2006) señala que las actitudes hacia el empleo difieren entre sociedades y grupos sociales, adquiriendo gran importancia los valores culturales que emanan de la idiosincrasia de cada lugar y más específicamente de los atributos de las personas que conforman cada territorio.

Diversos estudios abordaron la problemática del desempleo y su repercusión desde el ámbito de la
Psicología Social, como respuesta a la escasa importancia que los modelos económicos han otorgado a las variables psicosociales (Álvaro, 1992; Álvaro y Garrido, 2005; Buendía, 1990; García, 1993; Garrido, 1995; Garrido, 1996, Izquierdo, 2008). Esta escasa importancia que los modelos económicos otorgan a las variables psicosociales les hace perder de vista la influencia que los factores anteriores ejercen en los planes de acción de las personas desempleadas, lo que, a su vez, ha llevado al olvido de las importantes consecuencias psicosociales que el desempleo ocasiona en aquellas personas que lo sufren (Izquierdo, 2005). Estas consecuencias se hacen más explícitas en los grupos de mayor vulnerabilidad: un alto porcentaje de niños ven mermados sus derechos debido a la necesidad de incorporarse al mercado de trabajo, las mujeres sufren una inserción laboral menos favorable que la población masculina y no menos acuciada es la inserción laboral de las personas de mayor edad (Izquierdo, 2010). Concede este autor especial relevancia a la influencia del contexto cultural donde tiene lugar el fenómeno del desempleo proponiendo, entre sus conclusiones, la necesidad de adoptar una perspectiva transcultural, para poder extraer de mejor manera los mecanismos y procesos que hacen del desempleo una experiencia distinta en cada una de las personas afectadas. Este planteamiento coincide con la consideración de Bruner (1997) en tanto que el crecimiento humano individual no puede separarse de la cultura en que ocurre. Bruner propone tres razones a la hora de explicar la importancia de la cultura en el desarrollo de los sujetos: en primer lugar, la psicología humana no se puede construir basándose solo y exclusivamente en el individuo; en segundo lugar, la psicología al estar inmersa en una cultura ha de organizarse en torno a los procesos de construcción y utilización del significado que conectan el desarrollo humano con la cultura; y, en tercer lugar, resalta la importancia de lo que él denomina la psicología popular (creencias, ideas, o conceptos que hay en la sociedad). El trabajo se convierte, además de un dispositivo de carácter económico para la satisfacción de necesidades primarias y del intercambio de bienes y servicios, en un mecanismo para el establecimiento de la relaciones sociales interpersonales, un medio de participación cívica (como soporte de la integración sociopolítica y cultural), una fuente de bienestar subjetivo (como base para la integración de roles, estatus e identidad sociales), un contexto para socialización secundaria y un punto de articulación entre la vida pública y la privada, así como entre los proyectos individuales y los colectivos (Blanch, 2001).

\section{Escala de Necesidades, Culturas Nacionales y Regionales}

La condición humana continuamente hace esfuerzos para satisfacer sus necesidades organizadas en 
una serie de niveles con jerarquía de importancia (Maslow, 1970) y entre estas necesidades se encuentran las condiciones laborales derivadas de la organización del trabajo. Éstas se han convertido en uno de los fenómenos más estudiados en los últimos años. Schlemenson (2002) señala aspectos como el salario, la tarea y la posibilidad de realización personal que la organización brinda, las alternativas de desarrollo y carrera que se ofrecen, oportunidades de participar, el confort y la salubridad de los lugares del trabajo, consideradas como necesidades fisiológicas y de seguridad satisfechas en aquellas sociedades altamente desarrolladas. Este autor considera que estos aspectos han de ser objeto de estudios de tipo psicosocial y cultural.

Desde un plano cultural, Hollander (1982) añade que el hombre depende de la cultura para obtener una perspectiva y enfoque coherente de vida. Al aportar realidad social, el efecto psicológico esencial de la cultura es ejercer influencia sobre los miembros de una comunidad, induciéndolos a adoptar normas distintas de pensamiento y acción. En el plano laboral, Hollander (1995) destaca como esencial la idea de trabajo en equipo, como una relación de interdependencia en el líder y sus seguidores. Hofstede (1999) define la cultura como formas de pensar, sentir y actuar. Considera que las personas llevan dentro de sí varias capas de programación mental que corresponde a diferentes niveles de cultura. Los programas mentales para estos niveles no necesariamente guardan armonía entre sí y, a menudo, entran en conflicto: (1) Nivel nacional: corresponde al propio país. El concepto de nación se aplica a las unidades políticas en las que se divide el mundo y no se corresponde con la noción de sociedad. Las sociedades son formas de organización social desarrolladas orgánicamente y las naciones, por su parte, forman conjuntos desarrollados históricamente aunque se compongan de grupos claramente diferentes y con minorías menos integradas; (2) Nivel de clase social: se asocia con las oportunidades de educación y con la profesión u ocupación y (3) Nivel organizativo o corporativo: es para aquellas personas que tienen un empleo, según la manera en la que han sido socializados los empleados por su organización laboral. Hofstede considera las dimensiones de las culturas nacionales como un aspecto de una cultura que puede medirse en relación con otras culturas. Estas dimensiones juntas representan un modelo multidimensional de diferencias entre culturas nacionales. Además pone de manifiesto problemas comunes pero con soluciones que difieren de país en país en diversos parámetros o dimensiones.

Otra manera de representar las diferencias entre países es por medio de tipologías en vez de dimensiones. Una tipología describe una serie de tipos ideales, cada uno de ellos difícil de encontrar. Por ejemplo la división de países en cultura latinoamericana, anglosajona y asiática constituye una tipología. Si bien las tipologí- as son más fáciles de captar que las dimensiones siguen siendo problemáticas en investigación empírica. Los casos reales rara vez corresponden totalmente a un único tipo ideal. Por el contrario, con un modelo dimensional los casos pueden clasificarse siempre de manera inequívoca. En éste último, a partir de las puntuaciones de sus dimensiones los casos pueden ser ordenados posteriormente de manera empírica en grupos con puntuaciones similares. Esos grupos forman entonces una tipología empírica. En la práctica, las tipologías y los modelos pueden considerarse complementarios. Los modelos dimensionales son preferibles a efectos de investigación y las tipologías se utilizan más con fines didácticos.

De las cuatro dimensiones encontradas por Hofstede $(1984,2005)$, sobre las que se pueden clasificar las culturas de las naciones, dos de ellas son particularmente relevantes en las formas que funcionan los grupos y las personas: la distancia de poder que define la naturaleza de las relaciones entre subordinados y superiores, y el colectivismo-individualismo que refleja la interdependencia de los grupos frente a la independencia. Helmreich (2000) en una encuesta realizada en 26 países de los cinco continentes evidenció diferencias nacionales muy significativas en cuanto a las relaciones adecuadas entre líderes y seguidores, en el grupo contra la orientación individual, y en valores con respecto a la adhesión a normas y procedimientos. Estos datos coinciden con una investigación anterior de Helmreich (1984), donde reconsidera las cuatro dimensiones de la cultura de Hofstede en que clasifica a las naciones dado que dicho modelo ha probado ser conceptualmente relevante en el medio laboral. Cada una de las dimensiones muestra grandes diferencias al ser medidas a través de las naciones. Los países latinos reflejan una distancia más alta del poder y una aceptación del tipo de liderazgo más autocrático con desigual distribución del poder. En relación al individualismocolectivismo, los latinos reflejan un carácter colectivista, orientación que es más congruente con el concepto de trabajo en equipo y armonía grupal. Es más, los latinos serían más proclives a no observar procedimientos escritos y transgredir las reglas, pero más efectivos para desarrollar caminos alternativos en el afrontamiento de nuevas situaciones.

Estas características se manifiestan en el estudio de Alonso y Domínguez (2008) respecto a la cultura organizacional y su incidencia en el desempeño laboral, con trabajadores de empresas aerocomerciales argentinas (pilotos y técnicos de mantenimiento de aeronaves), hallando la preeminencia idiosincrática de la cultura latina con rasgos como la trasgresión a las normas, cierto nivel de omnipotencia o autoritarismo y, junto a ello, la capacidad creativa para adaptarse a situaciones no estándares o críticas. Así, las culturas profesionales y culturas organizacionales están fuertemente determinadas por las características idiosincrásicas de la cultura nacional que son propias de cada país, las que final- 
mente inciden en el desarrollo de determinadas conductas en el sujeto, mediatizada por la propia estructura de personalidad del mismo.

\section{Resiliencia, una nueva perspectiva}

El término resiliencia es definido por Grotberg (1995) como la capacidad humana para hacer frente a las adversidades de la vida, superarlas o incluso ser transformado por ellas. Ante la experiencia de transformación que sufren algunas personas por experiencias de adversidad, la resiliencia ayuda a los individuos no sólo a enfrentar las adversidades sino también a beneficiarse de las experiencias. En el área de desarrollo humano cabe un mayor énfasis en la importancia de promover el potencial humano en lugar de destacar el daño. Los factores que resultan protectores para los seres humanos, más allá de los efectos negativos de la adversidad, de acuerdo a Melillo y Suárez (2001) son los siguientes: introspección, independencia, capacidad de relacionarse, iniciativa, humor, creatividad, moralidad y autoestima consistente. Esos atributos o factores conforman, al operar integradamente, un sistema de protección que fortalece el análisis y la toma de decisiones, pero sobre todo crea una plataforma o un mapa para enfrentar la crisis que se enriquece permanentemente. Los factores protectores que contribuyen a la resiliencia se convierten en características de personalidad que determinan al individuo y a su ambiente social, ayudándolo a superar la adversidad, adaptándose activamente a la sociedad y teniendo una mejor calidad de vida $\mathrm{y}$, ente sentido, parece importante promover la resiliencia como una suerte de "vacuna" y generadora de inmunidad frente a las agresiones del medio ambiente sobre el equilibrio psicofísico. Es un modo práctico y aplicable justamente a las poblaciones desfavorecidas, como modo de generar un estado que amortigüe los efectos nocivos de las situaciones de riesgo y que les permita avanzar hacia un desarrollo saludable. Cómo conseguir esquivar el bache depende del grado de vulnerabilidad que tenga cada persona: experiencias laborales difíciles, desempleo, problemas económicos, pérdida de un familiar, etc. Nunes (2010) afirma que la experiencia adquirida durante el desarrollo es esencial para la construcción de un curso de vida satisfactorio.

El concepto de resiliencia y la posibilidad de su promoción tienen la virtud de una transdisciplinariedad fructífera entre el ámbito de lo social y lo psicológico y abren perspectivas de trabajo e investigación con un carácter preventivo, mucho antes de la emergencia de una patología o problema. Por tanto, la resiliencia es un concepto global y multifacético, asociado con numerosas características individuales y multisistemáticas. Se considera la promoción de la resiliencia como un proceso de consolidación y fortalecimiento de la instancia yoica del sujeto, que de ese modo se vuelve capaz de mediar los conflictos entre los deseos del sujeto, sus instancias críticas y la realidad. Los conflictos pueden ser más manejables para el yo resiliente. De acuerdo con Grotberg (2001), implica la promoción de determinados factores como "yo tengo", "yo soy", "yo estoy", "yo puedo", que permite prevenir, minimizar o sobreponerse a los efectos derivados de la adversidad, incluso anticiparse a adversidades inevitables.

En las dos últimas décadas se ha prestado especial atención a la resiliencia, que entraña la esperanza de una prevención satisfactoria. Si bien existen numerosos desarrollos teóricos e investigaciones científicas sobre la resiliencia, encontramos pocas evidencias en el contexto laboral. En la presente investigación, en virtud de los resultados obtenidos, se pretende incorporar los factores de protección de la resiliencia en situaciones de adversidad como la pérdida de un empleo, fundamentalmente en contextos de incertidumbre laboral.

Basándonos en lo señalado hasta este momento, el objetivo de este estudio es analizar la influencia de la cultura en la percepción sobre la situación de desempleo que sufren los hombres y mujeres mayores de 45 años de Paraguay usando una metodología cualitativa.

\section{Método}

\section{Participantes}

La investigación se realizó sobre una muestra de 162 personas desempleadas residentes en los asentamientos campesinos de la ciudad de Coronel Oviedo, capital del Departamento de Caaguazú (Paraguay). El total de la muestra, según el género, estaba formada por 97 hombres y 65 mujeres. Se constató un elevado número de personas con analfabetismo rural entre las personas entrevistadas. Los participantes fueron contactados en diversos lugares y, tras informales de los objetivos del estudio, se les invitó a responder a diversas preguntas, garantizándoles el anonimato y el empleo confidencial de las respuestas suministradas.

\section{Instrumento}

Se utilizó una entrevista semiestructurada, que por su flexibilidad permitió alterar el orden de las preguntas y la forma en que se formulaban, con el fin de recabar información en relación a la percepción sobre la situación de desempleo de la mencionada población de estudio. Con un carácter meramente descriptivo, mediante las entrevistas se obtienen una serie de información que ilustra, de propia voz, las experiencias propias de las personas desempleadas. En palabras de Buendía, Colás y Hernández (2003: 275) la entrevista nos permite "comprender las perspectivas y experiencias de las personas que son entrevistadas".

La entrevista fue adaptada para su aplicación con desempleados mayores de 45 años de Paraguay, previa 
comprobación de las condiciones científicas de fiabilidad y validez en el estudio de Izquierdo (2005). Se estructuró en torno a seis dimensiones, que conforman las escalas de actitudes hacia el trabajo. Cada escala, a su vez, disponía de una serie de cuestiones relacionadas con la búsqueda de empleo, con un total de 33 cuestiones a responder por las personas participantes en la investigación. Las escalas descritas son las siguientes:

1. Centralidad del empleo: se refiere a la importancia que los sujetos conceden al empleo, en tanto que valor instrumental y que valencia psicosocial, en las circunstancias contemporáneas de su vida. En el marco de la socialización de los individuos, la centralidad del empleo supone colocar al empleo como valor central de su vida.

2. Explicación del desempleo: Atribución interna y Atribución externa: explora en qué medida aparecen indicios del papel de tales atribuciones causales sobre las probabilidades de salida de ese estado. Se refiere a la explicación que el demandante de empleo da sobre su falta de trabajo.

3. Autoconcepto personal y profesional: engloba los resultados de una serie organizada de respuestas de los sujetos concernientes a quién, qué, cómo es y cuánto vale uno mismo, en tanto que persona y que profesional. Sobre esa síntesis de percepciones autorreferenciales basa el sujeto parte de su autoimagen, autoestima, autoevaluación, autopresentación y expectativas de desarrollo individual.

4. Disponibilidad para el empleo: examina la disponibilidad subjetiva al empleo por parte de individuos particulares. En tanto que actitud general, conlleva una representación de las ventajas e inconvenientes del estar empleado, los afectos asociados a tales percepciones y cierta orientación estratégica con respecto al mercado de trabajo.

5. Percepción subjetiva sobre la contratación de los empresarios: explora las percepciones que los desempleados tienen sobre aquellos aspectos que los empresarios valoran en su contratación. Un amplio número de las situaciones de discriminación en el ámbito laboral se producen por estereotipos y prejuicios acerca de los mayores de 45 años.

6. Estilo de búsqueda de empleo: estima el nivel de actividad con que un demandante de empleo desarrolla su vida cotidiana y su búsqueda de empleo. Aquí, a diferencia de las demás escalas, se le pide al demandante que indique si ha realizado recientemente la actividad que se le especifica.

Las entrevistas fueron grabadas en guaraní, que es el idioma predominante de los asentamientos campesinos del interior de Paraguay, lo que hizo posible que fuera una muestra verdaderamente autóctona, puesto que el guaraní constituye la lengua habitual de uso cotidiano. La recogida de información, al no existir una base de datos de personas desempleadas, se realizó en vías públicas, zonas de mayor confluencia (plazas, mercados, etc.) y en los propios hogares, siempre con la colaboración de un traductor. El número de personas que rehusaron fue muy pequeño, mostrando la población una actitud bastante favorable para participar en el estudio. La información obtenida fue transcrita en un procesador de textos y, posteriormente, analizada en el programa Ethnograph 5.0. aplicando las funciones de búsqueda selectiva, frecuencias y búsqueda de códigos múltiples, generándose un listado de ocho categorías correspondientes a cada una de las escalas de actitudes hacia el trabajo.

\section{Resultados}

Se establecieron ocho ejes de discusión, correspondientes a las categorías definidas, que expresan la valoración sobre la situación actual de los desempleados, su imaginario sobre las cualidades buscadas para la contratación por parte de los empleadores. El primer eje describe la situación actual de trabajo percibida por los entrevistados. El resto de ejes conforma cada una de las escalas de actitudes, con la salvedad de la escala explicación del desempleo que se subdivide en atribución interna (eje 3) y atribución externa (eje 4). Los ejes de discusión son los siguientes:

\section{Descripción de la situación de trabajo actual en Paraguay}

Los comentarios realizados por los participantes destacan la situación de "crisis económica", describiendo la carencia de puestos de trabajo en relación con épocas anteriores. Existe descontento por la actuación de Estado y de sus representantes señalando “.... porque acá en el Paraguay está yendo mucho la política, las elecciones internas, las nacionales, entonces ahí se gasta mucha plata..." Cabe señalar que el contexto sociopolítico paraguayo, en el momento de la aplicación del instrumento de recolección de información, está caracterizado por ser época de elecciones presidenciales en donde se hace cuenta de la gestión de los gobernantes como forma de captar votos.

\section{Importancia del trabajo}

Los entrevistados hacen referencia al trabajo como principal forma de sustento económico para cubrir sus necesidades mínimas, entre las que más sobresalen el acceso a educación y la salud. Los padres y madres de familia hacen constante hincapié en la relación educa- 
ción - trabajo, señalando que sin educación no es posible acceder a puestos de trabajo, sin embargo indican que esta relación tampoco actualmente es directa, puesto que varios de los hijos de las familias entrevistadas son profesionales y están desempleados. La salud y seguridad también fueron categorías analizadas, en menor grado que la educación, pero señalan que mediante el trabajo se puede acceder a servicios de salud y los ingresos económicos brindan seguridad familiar.

\section{Motivos por los cuales busca tener un trabajo}

En relación directa con el punto anterior, sobre la importancia de tener trabajo, los entrevistados señalan que buscan tener trabajo a fin de obtener ingresos para tener vivir, relacionando directamente, por lo general, trabajo con la satisfacción de las necesidades materiales. También para una fracción de los entrevistados, el trabajo, o mediante el trabajo se puede armonizar la convivencia familiar y personal, como forma de sentirse realizado: "Para mí que va a haber más unión de familia, que si todos tenemos lugar de trabajo, que hasta descansar va a tener más sentido, vamos a estar de vuelta a nuestra casa y vamos a compartir y seguramente si tiene un poco de exuberancia puede la familia darse más unida".

\section{Motivos por los cuales no se accede al trabajo asalariado}

Una de las características que resalta en este eje es la del Estado; el mayor porcentaje del gasto público del estado paraguayo va destinado al pago de salarios, desde épocas anteriores el Estado ha sido el mayor empleador, siendo reciente la apertura al mercado de competencia donde interviene con preponderancia el sector privado. Desde esta perspectiva, la mayoría de la población entrevistada señala que la causa del no acceso a puestos de trabajo se debe al Estado representado en la figura de sus gobernantes. "Porque está mal administrado el estado, los administradores con corruptos, todos los partidos son corruptos." Los participantes señalan la corrupción, en todas las esferas de la estructura social paraguaya, como una de las causas de carencia de acceso al trabajo. "Lo primero es que no tengo una profesión fija a no ser que sea un agricultor con su tierra, hay una limitación para poder conseguir trabajo". La educación o la falta de profesión es considerada como un factor limitante para acceder a puestos de trabajo, así lo señala una participante "Para las mujeres es más difícil conseguir trabajo asalariado, principalmente las mujeres se dedican a ser amas de casa y hay muy pocas oportunidades para poder desenvolverse profesionalmente”.

\section{Cualidades que podrá aportar como trabajador/a}

La población de estudio encontró cierta dificultad al interpretar esta cuestión, no ocurriendo así en el resto de preguntas de la entrevista, quizás debido a la diferencia en los códigos linguísticos. La mayoría de los participantes ha señalado como cualidad la capacidad de ejercer algún tipo de trabajo en general relacionado a su profesión, como por ejemplo, “... Albañil, carpintero, puede ser de todo un poco en esas ramas, pero no así profesionalmente por no tener estudios". No obstante se puede rescatar que han sido señaladas las cualidades de "persona responsable" en los emprendimientos a realizar.

\section{Trabajos por los cuales optaría realizar}

Los entrevistados han señalado que les gustaría ejercer una ocupación, si consiguen algo que tenga que ver con su experiencia será mejor que están dispuestos a adaptarse a los requerimientos de los contratantes. "Voy a tener que adaptarme a lo que yo pueda hacer, de acuerdo a mi capacidad y a mi preparación y de acuerdo a mi edad, todo depende de eso".

\section{Criterios de selección utilizados para la selección de trabajadores/as}

"Los patrones ahora tienen que buscar personal honesto porque todos los personales son corruptos ahora...”. La categoría conceptual más utilizada para describir el criterio de selección utilizado por los empleadores para la selección de trabajadores es el de la "confianza". Esto puede entenderse partiendo de la base del tipo de trabajo realizado por lo general por la población de estudio, en donde se recurre a referencia de otros empleadores. El uso del curriculum vitae u hoja de vida, puede suponerse que no es un instrumento comúnmente utilizado, considerando que no se cuenta con estudios "profesionales". "Aparte del profesionalismo lo que los patrones hoy día ven es la confianza, el conocerle a la persona, porque, por el tema de la inseguridad los patrones hoy día eligen a personas de confianza".

\section{Acciones realizadas para conseguir trabajo}

Esta sección puede ser analizada en dos sentidos, de acuerdo a las respuestas dadas. Por una parte se encuentran los entrevistados que actualmente ya no buscan tan arduamente un trabajo, y, los que opuestamente sí lo hacen. Se puede observar que existe una desesperanza en relación a las oportunidades para conseguir trabajo, los motivos han sido señalados anteriormente, "nada en especial", es una de las respuestas 
dadas, pero en general enmarcan que no hay fuentes de trabajo por lo que no han buscado (por lo menos en los últimos días). "No me dediqué luego a buscar porque me dedico a ser ama de casa y a lo que yo produzco acá, lo poquitito que haga que me mantiene...”. Sin embargo, también constatamos que existe una franja que recurre a todos los medios posibles a fin de conseguir por lo menos ganancias diarias a fin de cubrir los gastos diarios. "Un trabajo que me dedique a buscar más bien por cualquier trabajo para pode solventar los gastos...". "Anteriormente me dedicaba a buscar trabajo, hoy día ya no busco porque no hay, entonces me dedico espiritualmente a esparcir el evangelio de Dios". "Todos los días salimos a buscar trabajo, nos vamos a las desmotadoras, a las industrias, buscando puestos de trabajo pero actualmente no hay ni esperanzas, te dicen que si sale alguna persona seguro va a haber algún lugar, porque al salir alguna persona se hace casi sin ser trabajo. Siempre andamos buscando trabajo pero se hace muy difícil porque en nuestro caso que en desmotadoras, en industrias a pedir puestos de trabajo, ni siquiera esperanzas dan, porque no hay puestos de trabajo, te dicen que en caso de que salga alguna persona seguramente va a haber puestos de trabajo, pero eso casi nunca sucede porque nadie deja su trabajo, no hay puestos de trabajo aunque busquemos todos los días".

\section{Conclusiones}

La realización de esta investigación ha permitido no sólo conocer la influencia de la cultura en las actitudes que presentan los desempleados frente a la búsqueda de empleo sino también plantear algunas cuestiones que sería importante considerar al emprender futuras investigaciones en esta área. A pesar del interés que reviste esta cuestión, el estudio de las actitudes ha sido escaso en comparación con las investigaciones llevadas a cabo sobre otras consecuencias psicosociales derivadas de la falta de un puesto de trabajo (Izquierdo, 2008). El output arrojado, producto del análisis de la información usando Ethnograph, permitió extraer las siguientes conclusiones.

Las respuestas obtenidas en la entrevista destacan una alta centralidad personal del trabajo y una fuerte preocupación por la situación de desempleo en la que se encuentran. Esta importancia se manifiesta cuando se habla más en concreto de empleo o puesto de trabajo. Las personas entrevistadas manifiestan la importancia de tener un trabajo como un medio para la obtención de ingresos económicos con los que poder vivir. Por otro lado, los entrevistados comparten una nítida tendencia a la atribución interna de las causas del desempleo, reservando para los últimos lugares de una posible jerarquía causal las referidas a factores de tipo externo (causas externas). Las personas que realizan atribuciones de tipo interno tienen la posibilidad de analizar y resolver, en su caso, las causas que parecen ser las culpables de su situación de desempleo. Por el contrario, las soluciones para aquellas personas que atribuyen las causas de situación de desempleo a factores externos se tornan más complicadas.

Muchos de los entrevistados valoran muy positivamente su imagen personal. De igual modo, manifiestan una actitud muy favorable hacia su imagen profesional, aunque no tan acentuada como la anterior, al mostrar unos índices inferiores en su puntuación. Una valoración realista y positiva de estas características aumenta las posibilidades de encontrar empleo.

La mayoría de las personas entrevistadas afirman buscar empleo, aunque no todos lo hacen a cualquier precio ni incondicionalmente sino, en general, con determinadas condiciones económicas a las que se añade, en una parte significativa de los casos, también las temporales y las referidas a las características del puesto de trabajo. En la medida que una persona exprese estas limitaciones, disminuye considerablemente sus posibilidades de conseguir un empleo. De las entrevistas realizadas encontramos que quienes no buscan empleo se refugian en motivos de índole personal (dedicación a hijos menores, cuidado de familiares...) para encontrar una explicación razonable a tal conducta. Por el contrario, quienes si lo hacen declaran utilizar todos los medios posibles para encontrar trabajo. Con respecto a los procesos de selección, las entrevistas confirman que un amplio número de desempleados consideran una serie de características como determinantes de los procesos de selección. Así, asumir determinadas características resulta significativo para una predicción infructuosa de las posibilidades de inserción laboral. Estas características se convierten, en muchas ocasiones, en condicionantes de la selección de trabajadores por parte de los empresarios.

De la información analizada, si la comparamos con otros estudios llevados a cabo en otros países (véase Izquierdo, 2005), se aprecia que la cultura de cada país define ciertas formas de pensar, sentir y actuar conforme al planteamiento de Hofstede (1999). Podemos concluir, en relación a los supuestos de Bauder (2006), que las actitudes hacia el empleo difieren entre sociedades y grupos sociales, lo que determina que tales actitudes sean determinantes de una mayor o menor prolongación en situación de desempleo.

La realización de esta investigación ha permitido además la validación de un instrumento para analizar las actitudes que presentan los desempleados frente a la búsqueda de empleo. La metodología empleada en esta investigación puede ser útil para el análisis de las actitudes hacia el trabajo derivadas de la situación de desempleo en otros contextos y colectivos. Este estudio constituye, además, la base para una próxima investigación de carácter longitudinal, donde se analice de forma más precisa los resultados derivados de esta investigación, pudiendo llegar a una mayor clarificación de las relaciones entre desempleo y actitudes en la población 
desempleada. Por otro lado, diversos autores evidencian que la investigación sobre el desempleo ha prestado escasa atención a la influencia del contexto cultural en el que éste tiene lugar, por lo que es importante como señala Izquierdo (2008) la adaptación de una perspectiva transcultural que permita conocer los efectos del desempleo en otros contextos culturales.

Esta investigación tiene una serie de implicaciones prácticas para las organizaciones. En primer lugar, la ausencia de mecanismos apuntados por Blanch (2001) se evidencia aún más en determinados colectivos como los mayores de 45 años con poca preparación escolar al reducirse el abanico de puestos laborales a los que se puede aspirar. Al objeto de paliar este déficit de formación, se hace necesario que los diseños organizacionales echen una mirada a la cultura, subcultura, antecedentes demográficos y personalidad de los empleados (Triandis, 2002). Por otro lado, consideramos que estu- dios como éste, en los que se intenta describir la experiencia del desempleo, puedan servir, de alguna forma, para ofrecer soluciones y alternativas al problema del desempleo, así como plantear nuevos objetivos y contenidos para los programas de empleo que, a pesar de la complejidad del mundo laboral, es todavía un campo relativamente reciente. Estos programas, en virtud del análisis de la información obtenida en las entrevistas, han de incorporar los factores protectores de la resiliencia para superar situaciones de adversidad como la pérdida de empleo y, como destacan Melillo y Suárez (2001), enfrentarse y sobreponerse a estas situaciones. Por último, los programas de empleo deben incluir la intervención en actitudes conductuales de las personas desempleadas dado que, como recoge los estudios de Piqueras, Rodríguez y Rueda (2008) y Montilla (2005) la motivación incide de forma significa en la búsqueda de empleo.

\section{Extended Summary}

Culture of a society can be understood as a group of values situated along various dimensions, some of which appear to be universal (Oyserman, Kemmelmeir \& Coon, 2002) and people location in each of these dimensions describes its cultural pattern. There are few researches arguing that culture can influence in attitudes toward work of unemployed people through the development of a series of beliefs and behaviors associated with job search. Being workless becomes an obstacle to potential development of individuals, which prevent, in one form or another, people access to satisfaction of its psychological wellbeing. According to Nunes (2010), the gained maturity throughout personal life development is essential for building a positive lifestyle. According to the International Labor Organization, we are witnessing a rise in unemployment rates in Latin America, a symptom of the effects of an international crisis without precedents that darken economy and affect all countries in the region. This situation is evidenced in Paraguay where unemployment has grown in recent years. On one side, companies suffer significant technological changes such as information technology that require them to reduce the workforce and, on the other side, economic recession has been strong and prolonged which has left many people without work.

We propose as research objective the analysis of the influence that cultural values exert on attitudes toward work. Thus, this investigation will assess to evaluate relevance of cultural values as a moderator variable of the effects of unemployment in other contexts, up to date not explored. For this purpose, carried out a review of literature on psychosocial consequences of unemployment, the influence of national and regional cultures, and culminates with the concept: "resilience". Subsequently, describes an empirical study that allowed us to collect 162 interviews of unemployed people to analyze their attitudes toward job search.
Based in which was presented before, the aim of this study is to analyze the cultural influence on the perception of the unemployment who suffer men and women over 45 years of Paraguay. In this connection, the study uses a qualitative approach.

\section{Method}

\section{Participants}

The investigation was realized on a sample of 162 unemployed residents at rural settlements of Coronel Oviedo, capital city of Caaguazú Department (Paraguay). Rural settlements are characterized by its mobility as a survival strategy (study or work) and have as principal mean of subsistence the agriculture based on minimum consumption and production of small surpluses for sale. The total of the sample, based on gender was made up by 97 men and 67 women. Evidence showed a high percentage of illiteracy among persons interviewed from rural background in terms of educational level. The participants were contacted in their homes, and having explained the objectives of this study, they were invited to answer a serious of questions and were guaranteed complete privacy and confidentiality with their responses.

\section{Instrument}

We used a semi-structured interview allowing the flexibility to alter the order of questions and how they were formulated in order to gather information in relation to perception of unemployment situation on the abovementioned studied population. The interview was structured on a dimensional scale of six levels 
based on their attitudes about work. Each scale in turn contained a series of questions pertaining to job seeking of unemployed participants in the survey.

\section{Results}

There were established eight lines of discussion that express the rating of current situation of unemployed and his imagination on qualities wanted for recruitment by employers. Being presented the information, it was grouped according to each scale of attitude toward work. The comments made by participants would refer to the situation of "economical crisis" describing the lack of jobs in relation to previous periods. People were dissatisfied by the government performance and their representatives. One of the characteristics highlighted in fourth axe is the State; major percentage of Paraguayan State public expenditure is assigned to pay salaries. From previous periods the State has been the elder employer, being recently the opening to the market of competition where the private sector is preponderant. From this perspective, most of the interviewed population signaled the State as the cause of the no access to jobs. The population studied has not been able to comprehend the questions, maybe because of differences in linguistic codes. The majority has signaled as a quality the ability to exercise some type of work, in general related to their professions. The interviewees have signaled that they would like to execute an occupation or something related to their preparation. The most used conceptual category to describe the criterion of selection used by employers is "confidence". This can be seen on the basis of the type of work done in general by studied population, where it turns to other employers references. Last axis was analyzed in two ways, according to the answers. On one hand, the interviewees who are no longer looking for hard work and, on the other hand, those who are looking for that kind of job. One can see that there is a despair in relation to opportunities to get a job, reasons have been already identified.

\section{Discussion}

In the distinct phases of the analysis of data, we have been collecting those central aspects of research in connection to the objective. A first review of literature on that area has offered us some relevant theoretical conclusions. The study of attitudes has been small in comparison with the investigations carried out on other psychosocial consequences arising from the lack of employment (see Izquierdo, 2008). The result of data analysis using Ethnograph allowed drawing the following conclusions.

Results confirm a high centrality of "work" in personality and a strong worry over the unemployment situation in which they are immersed. This importance was manifest when speaking in concrete about employment or job. The results of our analysis show that a large number of the interviewees expressed the importance of having a job as a mean of subsistence. It notes that the individuals interviewed share a clear trend to internal attribution of unemployment causes, reserving for the last places as a possible causal hierarchy, those referring to factors of an external type (external causes). People performing attributions of an internal type have the possibility to analyze and resolve the causes that seem to be the reason of their unemployment situation. On the contrary, solutions for those people who attribute the causes of unemployment situation to external factors become more complicated. A great number of unemployed valued very positively their personal image, showing a very favorable attitude to his professional image, although not as marked as the previous case. A realistic and positive assessment of these characteristics increases the chances to find a job. Most of the interviewees affirm that they are looking for a job, although not all of them do it to any price nor unconditionally; but in general, with certain economic and temporary conditions. To the extent that a person expresses these limitations, reduce considerably his possibilities of getting an employment. Not to assume certain characteristics results significant for a successful job placement. Results confirm that a large number of unemployed considered a series of characteristics as determinants for the recruitment process. Those characteristics usually became to conditions for the selection of workers by employers. Job search of people over 45 years old is not particularly high. Although, even if not all the interviewees are looking for an employment, there is a large number of them that are expecting to find a job. The degree of effort made us know the chances of getting a job. As a conclusion drawn by the interviews, it's remarkable that those who do not look for work tried to hide behind their personal nature (dedication to minor children, taking care of members of the family...) to find a reasonable explanation for such behavior. On the contrary, those who are looking for a job declare using all possible means. Brief, all the factors described (assessment of work, attribution to internal causes, self concept, low availability for employment, lack of activity in the research of employment...) are attitudes that guarantee an extended stay in unemployment.

From the presented results, when compared with other studies conducted in other countries (see Izquierdo, 2005) shows that the culture of each country defines differing ways of thinking, feeling and acting in accordance with the approach of Hofstede (1999). We conclude, in relation to cases of Bauder (2006), that attitudes towards work differs between societies and social groups, which determines that such attitudes are determinants of greater or lesser term of 
unemployment.The methodology used in this investigation can be useful to evaluate certain attitudes arising from unemployment situation in other contexts. Besides, this study constitutes a basis for forthcoming longitudinal investigation where we could analyze more precisely the results achieve on this investigation. The lack of mechanisms aimed by Blanch (2001) is even more evident in certain groups such as people over 45 years with little school. In order to softening this training deficit, it's necessary that organizational structures have a look to culture, subculture, demographical background and personality of applicants (Triandis, 2002).

Diverse authors have shown that investigation about unemployment has paid scarce attention to cultural context influence. So, as Izquierdo pointed out (2008), it's significant the adaptation of a cross-cultural perspective which allow us to know the effects of unemployment in specific cultural contexts. Describing the experience of unemployment can serve, somehow, to offer solutions and alternatives to this problem. Also, it provides new goals and contents to reach in employment programs that despites complexity of the labor world is still relatively recent. These programs must incorporate the protective factors of resilience to overcome adversity situations such as loss of employment, as emphasized Melillo and Suarez (2001), confront and overcome these situations. Finally, employment programs should include intervention in behavioral attitudes of unemployed people since motivation affects significantly in job search, as the studies of Piqueras, Rodríguez and Rueda (2008), and Montilla (2005) collects.

\section{Referencias}

Alonso, H. J. y Domínguez, D. (2008). La cultura organizacional en aeronáutica civil. Actas de las IX Jornadas de Psicología de las Organizaciones y el Trabajo. Montevideo, Uruguay.

Álvaro, J. L. (1992). Desempleo y bienestar psicológico. Siglo XXI: Madrid.

Álvaro, J.L. y Garrido, A. (2005). Desempleo, salud y exclusión social. Red Social Interactiva, 6. En http://redsirevista.cebs-es.org/index.asp? IdRev $=16$.

Bauder, H. (2006). Origin, employment status and attitudes towards work: immigrants in Vancouver, Canada. Work Employement and Society, 20, 709-729.

Blanch, J. M. (2001). Empleo y desempleo: ¿viejos conceptos en nuevos contextos?. En E. Agulló y A. Ovejero (Coords.), Trabajo, individuo y sociedad. Madrid: Pirámide.

Blanch, J. M. (2007). Psicología Social del Trabajo. En Aguilar, M. y Reid, A. (Coords.). Tratado de Psicología Social. Perspectivas Socioculturales. México: Anthropos.

Blanch, J. M. y Cantera, L. M. (2009). El malestar en el empleo temporal involuntario. Revista de Psicología del Trabajo y de las Organizaciones, 25, 59-70.

Bruner, J. (1997). La educación, puerta de la cultura. Madrid: Visor.

Buendía, J. (1990). Psicopatología del desempleo. Anales de Psicología, 6, 1, 21-36.

Buendía, L., Colás, P. y Hernández, F. (2003). Métodos de investigación en psicopedagogía. Madrid: McGraw-Hill.

García, A. M. y García, M. G. (2008). La influencia de los rasgos psicológicos en las actitudes hacia el empleo. Revista de Psicología del Trabajo y de las Organizaciones, 24, 203-233.

García, Y. (1993). Desempleo: alteraciones psicológicas. Valencia: Promolibro.

Garrido, A. (1996). Psicología social del desempleo. En J. L. Alvaro, A. Garrido y J. L. Torregrosa (Coords), Psicología social aplicada. Madrid: McGraw-Hill.

Garrido, A. (2006). Sociopsicología del Trabajo. Barcelona: UOC.

Garrido, M. (1995). Desempleo y psicopatología. Un estudio empírico e interpretación psicodinámica. Valencia: Promolibro.

Grotberg, E. (1995). Fortaleciendo el espíritu humano. La Haya: Fundación Bernard Van Leer.

Grotberg, E. (2001). Nuevas tendencias en resiliencia. En Melillo, A. y Suárez, E. (comp.). Resilencia. Descubriendo las propias fortalezas. Buenos Aires: Paidós.

Helmreich R. L. (1984). Cockpit Management Attitudes. Human Factors, 26, 583-589.

Helmreich, R. L. (2000). Culture and error in space: Implications from analog environments. Aviation, Space, and Environmental Medicine, 71, 133-139.

Hofstede, G. (1984). Culture's Consequences: International Differences and WorkRelated Values. Londres: Sage.

Hofstede, G. (1999). Culturas y organizaciones. El software mental. La cooperación internacional y su importancia para la supervivencia. Madrid: Alianza Editorial.

Hofstede, G. J. (2005). Cultures and Organizations: Software of the Mind. New York: McGraw-Hill.

Hollander, E. P. (1982). Principios y métodos en psicología social. Buenos Aires: Amorrortu.

Hollander, E. (1995). Ethical challenges in the leader-follower relationship. Business Ethics Quaterly, 5, 54-65.

Izquierdo, T. (2005). Actitudes hacia el trabajo de los desempleados mayores de 45 años. Granada: EUG.

Izquierdo, T. (2007). Cultura del desempleo en América del Sur. El caso concreto de los desocupados mayores de 45 años del departamento de Caaguazú (Paraguay). Actas del II Congreso Internacional de Educación en Valores. Camagüey, Cuba.

Izquierdo, T. (2008). El desempleo en los mayores de 45 años. Jaén: CES.

Izquierdo, T. (2010). Los nuevos retos del mercado laboral: una perspectiva desde la orientación profesional. Úbeda: Amarantos.

Maslow, A. H. (1970). Motivation and personality. New York: Harper.

Montilla, S. (2005). Diferencias individuales de los desem- 
pleados y constructos motivacionales: una relación de interdependencia predictora del desempleo estructural. Revista de Psicología del Trabajo y de las Organizaciones, 21, 269-298.

Melillo, A. y Suárez, E. (2001). Resiliencia. Descubriendo las propias fortalezas. Buenos Aires: Paidós.

Nunes, I. (2010). Motivational dynamics in the development of career attitudes among adolescents. Journal of Vocational Behavior, 76, 170-177.

OIT (2009). Panorama laboral 2009. América Latina y el Caribe. Lima: Oficina regional para América Latina y el Caribe.
Oyserman, D., Kemmelmeier, M. y Coon, H. (2002). Cultural psychology, a new look. Psychological Bulletin, 128, 110-117.

Piqueras, R., Rodríguez, A. y Rueda, C. (2008). Expectativas y duración del desempleo. Revista de Psicología del Trabajo y de las Organizaciones, 24, 129-151.

Schlemenson, A. (2002). La estrategia del talento. Buenos Aires: Paidós.

Triandis, H. C. (2002). Motivation to work in cross-cultural perspective. En J. M. Brett y F. Drasgow (Eds.), The psychology of work: theoretically based empirical research. Mahwah: Lawrence Erlbaum Associates.

Manuscrito Recibido: 21/10/2009

Revisión Recibida: 25/04/2010

Aceptado: 28/05/2010 